\title{
Revisión del género Boletus L. (Boletales, Basidiomycota) en Chile
}

(Review of the genus Boletus L. (Boletales, Basidiomycota) in Chile)

\author{
Riquelme C. ${ }^{1}$, Dibán MJ. ${ }^{2,3,4}$, Salazar-Vidal V. ${ }^{1,2 *}$ \\ ${ }^{1}$ Laboratorio de Química de Productos Naturales, \\ Facultad de Ciencias Naturales y Oceanográficas, \\ Universidad de Concepción. Concepción, Chile. \\ ${ }^{2}$ ONG Micófilos. Región del Biobío, Chile. \\ ${ }^{3}$ Laboratorio de Paleoecología. Facultad de Ciencias, \\ Universidad de Chile. \\ ${ }^{4}$ Instituto de Ecología y Biodiversidad. Ñuñoa, \\ Santiago, Región Metropolitana, Chile \\ *Autor para correspondencia: vivi.sal.vidal@gmail.com
}

RECIBIDO: 06 de Junio de 2019 APROBADO: 27 de Junio de 2019

DOI: 10.22370/bolmicol.2019.34.1.1754

LOS AUTORES DECLARAN NO TENER CONFLICTO DE INTERESES

Palabras claves: Butyriboletus, clave, taxonomía, morfología, distribución.

Key words: Butyriboletus, key, taxonomy, morphology, distribution.

\section{RESUMEN}

El género Boletus cuenta con alrededor de 300 especies, las cuales se encuentran ampliamente distribuidas en el mundo, formando micorrizas con diversos árboles. En Chile se han documentado seis especies: Boletus loyo, $B$. loyita, $B$. chilensis, B. putidus, B. bresinskyanus y B. araucariae, asociadas exclusivamente a Nothofagus spp., siendo Boletus loyo actualmente clasificada en el género Butyriboletus. En esta revisión, se describen los caracteres macro y microscópicos junto con la distribución climática de las seis especies nativas presentes en nuestro país de acuer- do a la literatura. Además, se presenta una clave dicotómica basada en bibliografía relevante para ayudar a su determinación.

\section{ABSTRACT}

The genus Boletus has around 300 species, which are distributed throughout the world, forming mycorrhizae with different trees. In Chile six species have been documented: Boletus loyo, B. loyita, B. chilensis, B. putidus, B. bresinskyanus and $B$. araucariae, which belong to Nothofagus spp., being Boletus loyo currently classified in the genus Butyriboletus. In this re- 
view, the macro and microscopic characters and climatic distribution of the six native species present in our country are described according to the literature. In addition, a dichotomous key based on relevant literature is presented to aid in its determination.

\section{INTRODUCCION}

El género Boletus L. pertenece a la familia Boletaceae y está compuesto aproximadamente por 300 especies (Kirk et al., 2008). En general, corresponden a especies ectomicorrícicas, las cuales están ampliamente distribuidas. Tienen gran importancia ecológica y económica debido a su comestibilidad (Singer, 1986; Hall et al., 1998). Muchos micólogos han contribuido al estudio taxonómico del género Boletus s.l. que pertenece a la familia Boletaceae, actualmente aceptada o dividida en subgéneros pequeños, secciones o diferentes géneros (Singer 1965, 1967, 1986; Watling, 1970; Smith \& Thiers, 1971; Corner, 1972; Horak, 1977, 2005, 2011).

El género Boletus se caracteriza por presentar basidiomas terrestres, carnosos, con un himenóforo poroide y en su mayoría con un estípite central (Murrill, 1909; Horak 1977; Dentinger et al., 2010). Macroscópicamente, se diferencia de los políporos, debido a su consistencia suave, hábito terrestre, himenóforo y pileipelis fáciles de desprender del píleo (Piepenbring, 2015). El análisis microscópico es requerido para su determinación, al igual que para todos los hongos, siendo importante considerar la morfología de las esporas y la pileipelis, junto con los cistidios en los tubos y en el estípite.

Horak (1977) puntualiza una relación inversa existente entre la diversidad ecológica de los bosques templados de Chile en conjunto con su alta productividad (Salas et al., 2006) y la es- casa cantidad de especies nativas conocidas de la familia Boletaceae. Solamente han sido descritas seis especies del género Boletus para la zona de distribución de los Nothofagus chilenos (Garrido, 1988; Palfner, 2005), específicamente, en un área restringida ubicada entre la Región del Maule y la Región de los Lagos. Estas especies son: B. loyo, B. loyita, B. putidus, B. chilensis, $B$. bresinskyanus y $B$. araucariae, siendo Nothofagus obliqua (Mirb.) Oerst un aliado fundamental para el establecimiento de estos hongos ectomicorrícicos (Horak, 1977; Garrido, 1985). Recientemente, Boletus loyo fue transferido al género Butyriboletus; sin embargo, esto aún se encuentra en discusión (Marín, 2018), por lo que para fines de esta revisión bibliográfica será considerado dentro del género Boletus.

\section{MATERIALES Y MÉTODOS}

En este trabajo, se revisó literatura relevante sobre el género Boletus en Chile, como por ejemplo, Horak (1977), Garrido (1988), Valenzuela et al., (1998), Palfner (2005) y las fichas de Clasificación de Especies publicadas en el Ministerio del Medio Ambiente. Además, se analizaron las condiciones climáticas y altitudinales bajo las cuales crecen las especies de Boletus en Chile, utilizando los datos de la plataforma virtual World Clim (Hijmans et al., 2005)

\section{Clave dicotómica}

1. Píleo marrón rojizo, rojo a púrpura................2

1’. Píleo de tonalidades marrones sin tinte rojizo . .5

2. Esporas de 11 a $17 \mu \mathrm{m}$

Boletus loyo (sin. Butyriboletus loyo)

2'.Esporas de hasta $13 \mu \mathrm{m} \ldots \ldots \ldots \ldots \ldots \ldots \ldots \ldots \ldots$ 
3. Píleo marrón-amarillento a marrón-rojizo, con cistidios cilíndricos a claviformes de 25-50 x $5-8 \mu \mathrm{m}$ Boletus chilensis

3'. Píleo rojo oscuro, purpúreo o vináceo, con cistidios fusiformes...................................4

4. Tubos de hasta $13 \mathrm{~mm}$ de largo, y hasta 1,5 mm de diámetro. Cistidios de 47-65 x 7-10 $\mu \mathrm{m}$ hialinos con un ligero color marrón ocre.

\section{Boletus bresinskyanus}

4'. Tubos de hasta $8 \mathrm{~mm}$ de largo, y hasta $2 \mathrm{~mm}$ de diámetro. Cistidios de 35-50 x 9-12 $\mu \mathrm{m}$ hialinos

Boletus loyita

5. Estípite con tonalidades ocráceas, con el ápice amarillento, de 50-60 × 25-50 mm, esporas de $8,5-15 \times 4.5-6 \mu \mathrm{m}$ Boletus araucariae

5'. Estípite con tonalidades castañas, de 50$100 \times 15-40 \mathrm{~mm}$, esporas de 8-10,5 x 3-4 $\mu \mathrm{m}$ Boletus putidus

Boletus loyo Phillippi (Fig.1) (Sinónimos: Boletus loyus Espinosa, Butyriboletus loyo (Phillippi) Mikšík)

Descripción: Píleo de 60-350 $\mathrm{mm}$ de ancho, cóncavo hasta plano, pulviniforme, liso, seco, glutinoso cuando húmedo, rojo oscuro, contexto amarillo, tornándose celeste en cortes o heridas. Tubos de 6 a $20 \mathrm{~mm}$ de largo, y hasta $1 \mathrm{~mm}$ en diámetro, amarillos con tono oliváceo. Estípite 80-150 x 40-70 mm, céntrico, anchamente claviforme hasta bulboso, amarillo con base roja. Esporas fusiformes, 11-17 x 4-6 $\mu \mathrm{m}$, amarillentas, lisas, no amiloides. Basidios 28-30 x 10-11 $\mu \mathrm{m}$ (modificado según Horak, 1977 y Garrido, 1988). Cistidios 30-60 × 5-10 $\mu \mathrm{m}$, fusiformes. Caulocistidios 40-80 x 11-19 $\mu \mathrm{m}$. Es una especie inconfundible por su tamaño y sus colores característicos.
Hábitat y ecología: Forma asociaciones ectomicorrícicas con $N$. obliqua y $N$. dombeyi (Mirb.) Oerst. (Horak, 1977; Garrido, 1988). También se registran observaciones bajo $N$. alpina (Poepp. \& Endl.) Oerst (Horak, 1977) y bajo N. glauca (Phil.) Krasser (Donoso, 1990). Los basidiomas crecen de manera solitaria o en pequeños grupos en el suelo, desde el nivel del mar hasta los 1.300 $\mathrm{m}$ aproximadamente. Se observa más frecuentemente en bosques de Coihue-Roble.

Distribución geográfica: Con registros entre la Región del Maule y la Región de Los Lagos.

Comentarios: El nombre común de Boletus loyo es "Loyo" y es una especie muy apetecida, ampliamente recolectada para consumo y para la venta en ferias locales, debido a su exquisito sabor y consistencia. Asimismo, B. loyo es la especie de Boletus más grande registrada en nuestro país, cuyo píleo en ocasiones puede superar los $30 \mathrm{~cm}$ de diámetro.

\section{Categoría de conservación vigente en Chile: EN PELIGRO (EN).}

\section{Boletus loyita E. Horak (Fig. 2)}

Descripción: Píleo de 25-60 mm de diámetro, hemisférico, levemente deprimido en el centro, rojo oscuro a púrpura en basidiomas jóvenes tornándose después marrón rojizo oscuro, seco, velutinoso o minuciosamente fibriloso. Tubos deprimidos o emarginados a subdecurrentes en la zona de contacto con el estípite hasta $8 \mathrm{~mm}$ de largo y $2 \mathrm{~mm}$ de diámetro, poligonales, de color amarillo intenso, tornándose dorado amarillento en carpóforos envejecidos. Estípite de 40-100 x 10-20 mm, subfusiforme a cilíndrico, usualmente rojizo oscuro a púrpura, seco minuciosamente pruinoso, reticulación ausente, sólido. Contexto amarillo en el píleo y en la parte superior del estípite, se torna inmediatamente azulado en cortes 
frescos, rojo púrpura en la base del estípite. Esporas fusiformes, $10-11 \times 3,5-4(4,5) \mu \mathrm{m}$, amarillentas marrones, lisas. Basidios 25-32 x 8-10 $\mu \mathrm{m}$. Cistidios 35-50 x 9-12 $\mu \mathrm{m}$, fusiformes, hialinos, de pared delgada. Reacciones químicas, $\mathrm{KOH}$ amarillo en el píleo, negativa en el contexto. Sabor y olor no distintivo (modificado según Horak, 1977 y Garrido, 1985).

Hábitat y ecología: Basidiomas crecen en el suelo, asociándose en simbiosis ectomicorrícica con N. dombeyi y N. obliqua (Horak, 1977; Garrido, 1985). También, se ha reportado en asociación con $N$. alessandrii Espinosa, $N$. dombeyi, $N$. alpina y $N$. glauca.
Distribución geográfica: Distribución conocida entre las Regiones del Maule y de los Ríos.

Comentarios: Es una especie más pequeña que $B$. loyo, el nombre común que recibe $B$. loyita es "Pichi loyo" que alude a que tiene un tamaño más pequeño que el "Loyo". Es un hongo muy apreciado como comestible, pero que según recolectores locales en las zonas donde crece, ha disminuido drásticamente su población en los últimos 10 años.

Categoría de conservación vigente en Chile: VULNERABLE (VU).

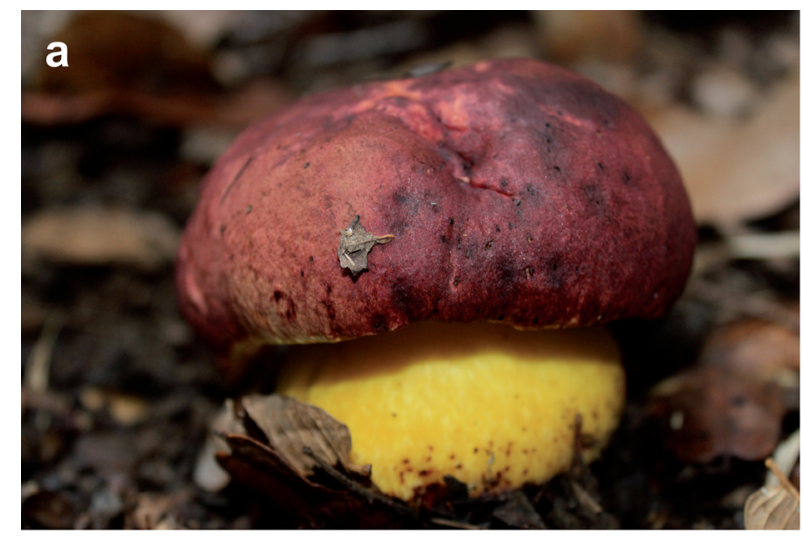

b

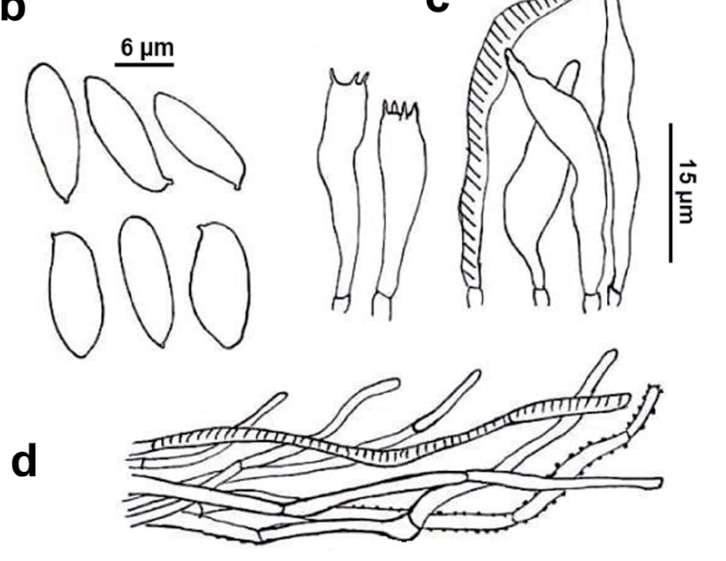

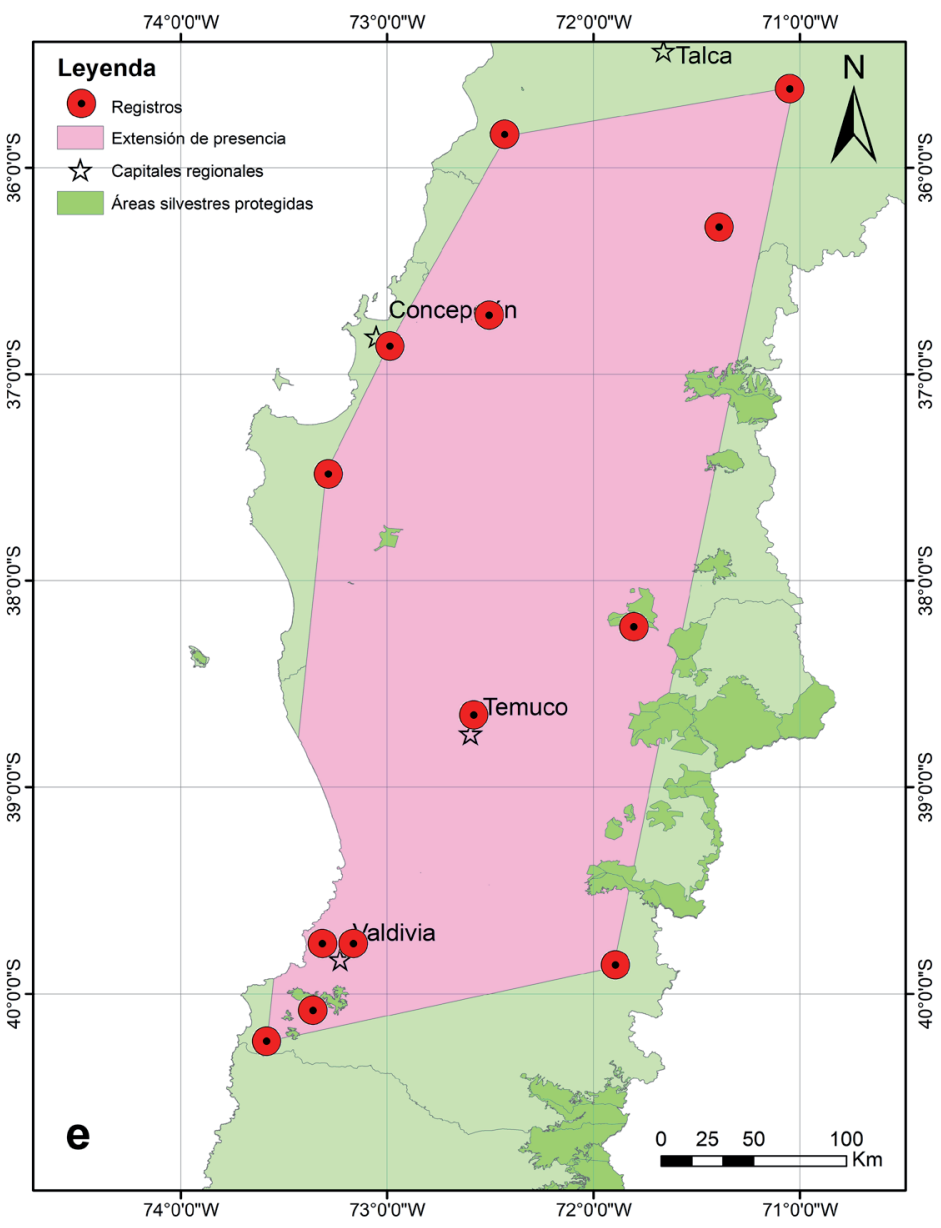

Figura 1. Boletus loyo. a. Basidioma juvenil (Fuente:Viviana Salazar); b. Basidiosporas; c. Basidios y queilocistidios (adaptados de Horak, 1977); d. Cutícula; e. Mapa de la distribución actual de B. loyo. 

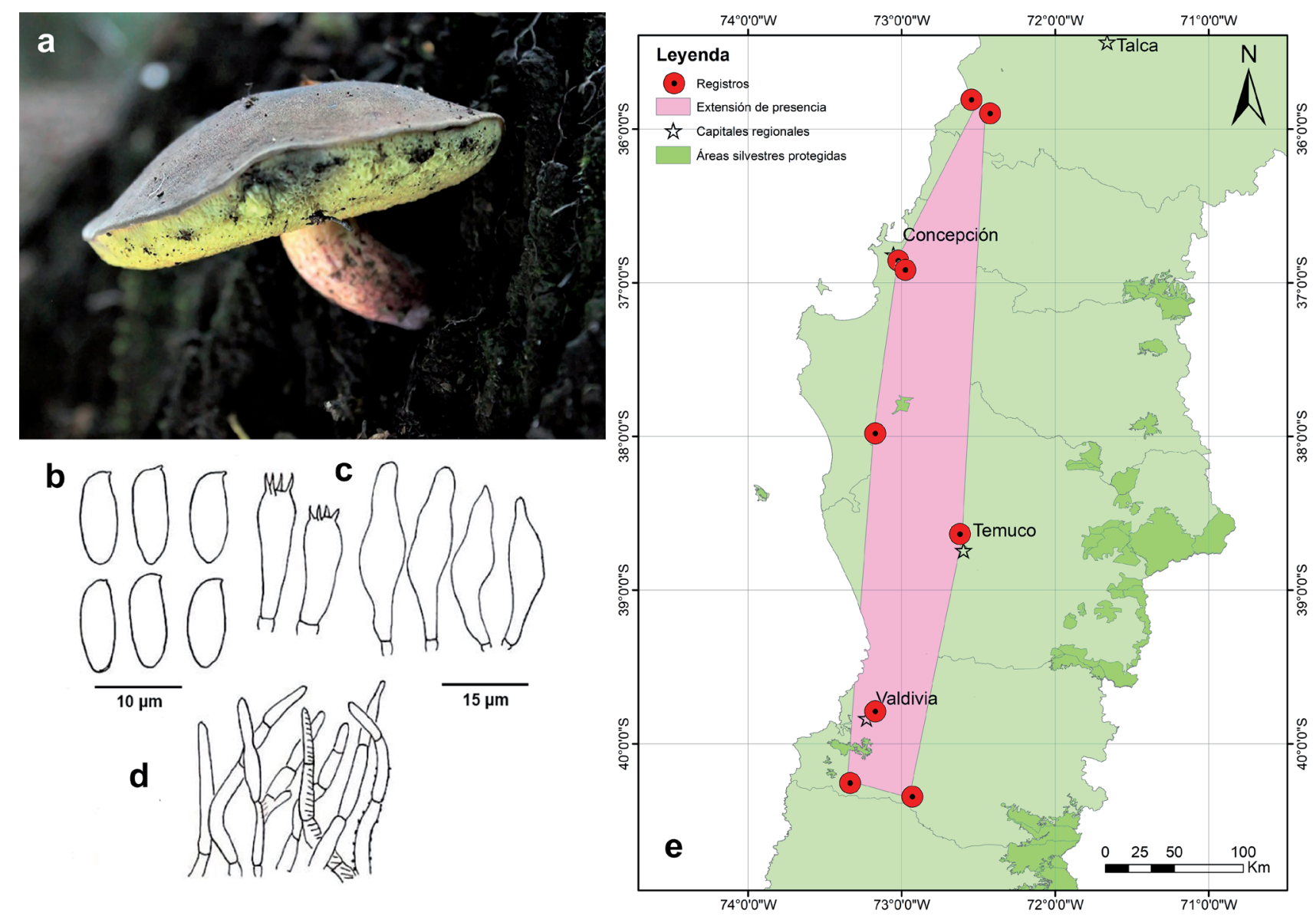

Figura 2. Boletus loyita. a. Basidioma adulto (Fuente: Johan Andrianoff); b. Basidiosporas; c. Basidios y queilocistidios (adaptados de Horak, 1977); d. Cutícula; e. Mapa de la distribución actual de B. loyita.

\section{Boletus chilensis Singer (Fig. 3)}

Descripción: Píleo de 80-140 mm de diámetro, convexo a pulvinado, finalmente deprimido en el centro, marrón amarillento a marrón rojizo, viscoso cuando húmedo, al secarse velutinoso o aterciopelado. Tubos hasta $15 \mathrm{~mm}$ de largo, hasta $1,5 \mathrm{~mm}$ de diámetro, de color amarillento a ocre, bocas de los tupos tornándose marrón rojizo en carpóforos maduros. Estípite de 40-100 mm de largo, 30-40 $\mathrm{mm}$ de ancho (ápice), atenuado hacia la base, algo radicante, robusto, amarillo en el ápice, rojizo a marrón en la base, de color similar al del píleo o más pálido desde la porción medial hasta la base, reticulado en la parte superior, seco, glabro y sólido. Contexto blanco, a veces rojizo en la parte superior del estípite, amarillo en la base, tornándose azulado en cortes. Esporas fu- siformes, 8-11 x 3,5-4 $\mu \mathrm{m}$, amarillentas, lisas, de pared delgada, sin poro germinal. Basidios 30-40 x 7-10 $\mu \mathrm{m}$. Cistidios 25-50 x 5-8 $\mu \mathrm{m}$, cilíndricos o claviformes. Reacciones químicas, $\mathrm{KOH}$ negativa o algo rojizo en el píleo. Sabor y olor no distintivo (modificado según Horak, 1977 y Garrido, 1988).

Hábitat y ecología: Basidiomas solitarios, a veces en grupos, que crecen formando asociación ectomicorrícica con N. obliqua y N. dombeyi (Horak, 1977; Garrido, 1985; Valenzuela et al., 1998).

Distribución geográfica: Hasta la fecha solamente existen registros entre la Región de la Araucanía y la Región de Los Lagos.

\section{Categoría de conservación vigente en Chile: EN PELIGRO (EN).}



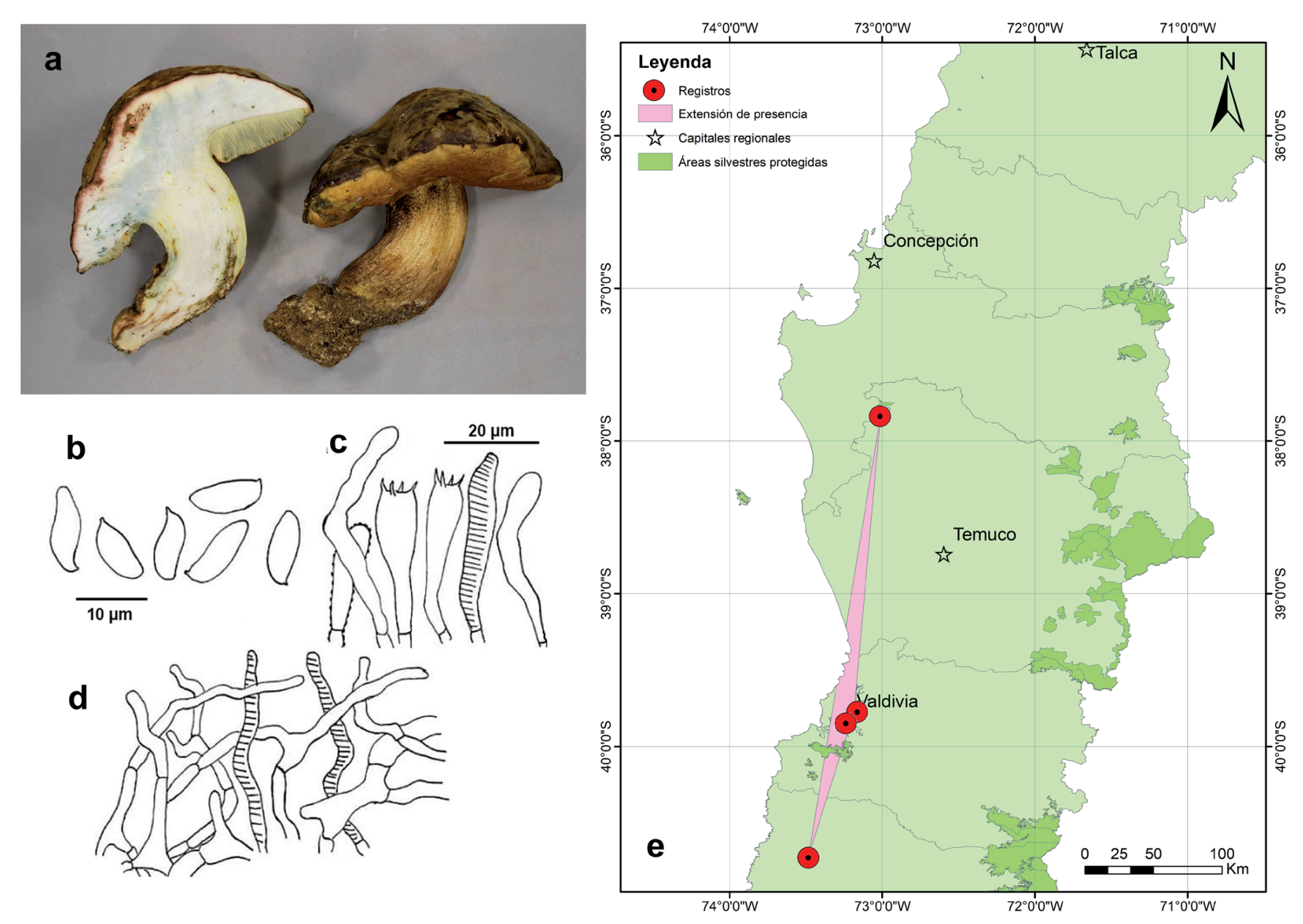

Figura 3. Boletus chilensis. a. Basidioma adulto (Fuente: Götz Palfner, MMA); b. Basidiosporas, c. Basidios y queilocistidios (adaptados de Horak, 1977), d. Cutícula; e. Mapa de la distribución actual de B. chilensis.

Boletus putidus E. Horak (Fig. 4) (Sinónimo: Tylopilus temucensis Palfner)

Descripción: Píleo hasta 110 mm de diámetro, en basidiomas jóvenes hemisférico volviéndose plano a deprimido al madurar, de color pardo, seco, velutinoso, algo viscoso en condiciones húmedas, margen ocasionalmente sobresaliendo, membranoso. Tubos emarginado-deprimidos en zona de contacto con el estípite, hasta $15 \mathrm{~mm}$ de largo, de color amarillo cuando joven, tornándose amarillo oliváceo a marrón oliváceo, bocas de los tubos poligonales, con tintes rosados al apretar se tornan levemente azuladas. Estípite de 50-100 x
15-40 mm, claviforme o fusiforme, con la base atenuada, ápice amarillento, base marrón rojizo o rojo cobre, seco, velutinoso o pruinoso, sin rastros de retículo, sólido. Contexto del píleo y del estípite blancuzco, tornándose granate pálido o marrón rosáceo en cortes frescos, porción superior del estípite amarillo, levemente cianescente. Esporas fusiformes, 8-10,5 x 3-4 $\mu \mathrm{m}$, amarillentas, lisas, de pared delgada. Basidios 30-36 x 7-10 $\mu \mathrm{m}$, tetraspóricos. Cistidios 35-70 x 5-8 $\mu \mathrm{m}$, fusiformes, hialinos, de pared delgada. Reacciones químicas, con $\mathrm{KOH}$ anaranjado en el píleo, negativa en el contexto e himenio. Sabor y olor algo agrio (modificado de Garrido, 1988). 
Hábitat y ecología: Forma asociaciones ectomicorrícicas con Nothofagus spp., particularmente, N. dombeyi y N. obliqua (Horak, 1977; Garrido, 1985), encontrándose los basidiomas sobre el suelo, de forma solitaria o en pequeños grupos de dos a tres ejemplares.
Distribución geográfica: Sólo existen registros entre las Regiones del Maule y de Los Ríos.

\section{Categoría de conservación vigente en Chile:} CASI AMENAZADA (NT).
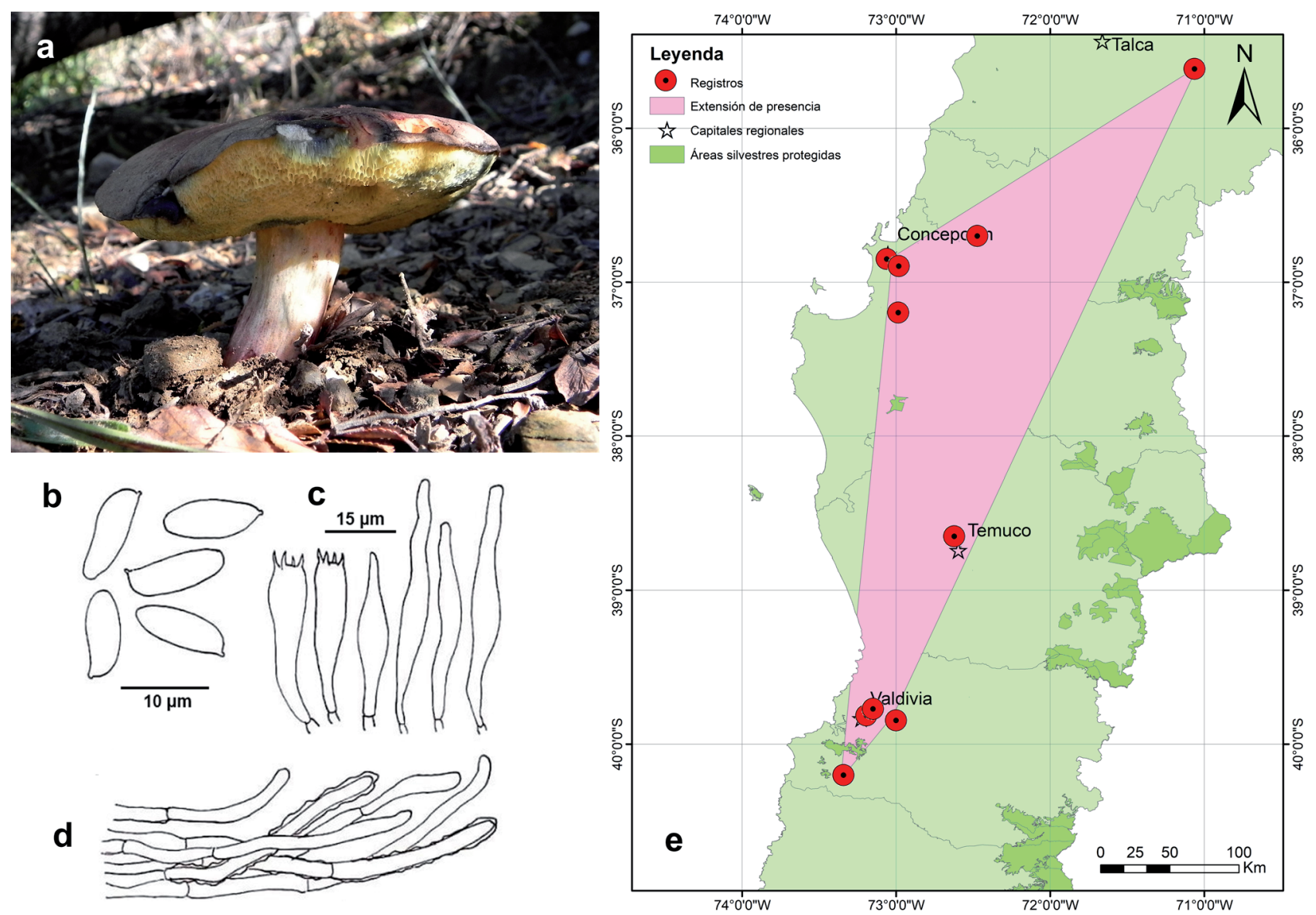

Figura 4. Boletus putidus. a. Basidioma juvenil (Fuente: Eitel Thielemann); b. Basidiosporas; c. Basidios y queilocistidios (adaptados de Horak, 1977); d. Cutícula; e. Mapa de la distribución actual de B. putidus.

\section{Boletus bresinskyanus Garrido (Fig. 5)}

Descripción: Píleo de 47-115 mm diámetro, hemiesférico, de plano a deprimido, finamente aislado, viscoso, vináceo cuando joven, luego granate claro, amarillo, azul cuando se toca; cutícula no extraíble. Tubos 4-13 mm de largo, anexos-emarginados, poligonales, anchos, 1-1.5 mm de diámetro, amarillo dorado, que se vuelven azules cuando se tocan. Estípite 47-84 x 9-14 mm, cilíndrico, en el ápice vináceo a rojo carmesí, azulado al tacto, base blanquecina, a rojizo carmesí muy profundo en la base, azulado al tacto, con micelio blanco en la base. Olor indistinto: Sabor ligeramente agrio. Reacciones químicas: poros al aplicar $\mathrm{KOH}$ marón rojizo cuando son jóvenes, luego rubí oscuro al envejecer. Esporas 9-13 x 4-6 $\mu \mathrm{m}$, fusiformes, lisas, con depresión supra-apicular, ausencia de poro germinativo, de color ocre claro parduzco. Basidios 27-42 x 7-11 $\mu \mathrm{m}, 4$ esporas. Queilo y 
pleurocistidios $47-65 \times 6-10 \mu \mathrm{m}$, fusiformes, hialinos con un ligero color marrón ocre. Cutícula del píleo con hifas cilíndricas de 2-15 $\mu \mathrm{m}$ de ancho, claramente de color marrón rojizo, con pigmentos membranosos o incrustantes. Hifas terminales cilíndricas o atenuantes hacia la punta (modificado según Garrido, 1988).

Hábitat y ecología: Forma asociaciones ectomicorrícicas con N. obliqua, y N. dombeyi donde ha sido reportado en bosques dominados por Weinmannia trichosperma Cav., Cryptocarya alba (Molina) Looser, Chusquea quila Kunth como especies acompañantes (Garrido, 1988).

Distribución geográfica: Con registros ubicados únicamente en la Región del Biobío, en la Cordillera de Nahuelbuta.

Comentarios: $B$. bresinskyanus muestra algunas similitudes $\operatorname{con} B$. loyita y $B$. araucariae. Se diferencia de $B$. loyita por presentar el píleo lubricado, las esporas más grandes y micelio basal blanco; de B. araucariae por hifas consistentemente cilíndricas en la cutícula del píleo, el estípite cilíndrico y el basidiocarpo más pequeño. Importancia económica: Comestible, a menudo consumido por los pueblos originarios, habitantes de Contulmo y sus alrededores.

\section{Categoría de conservación vigente en Chile:} NO EVALUADA.
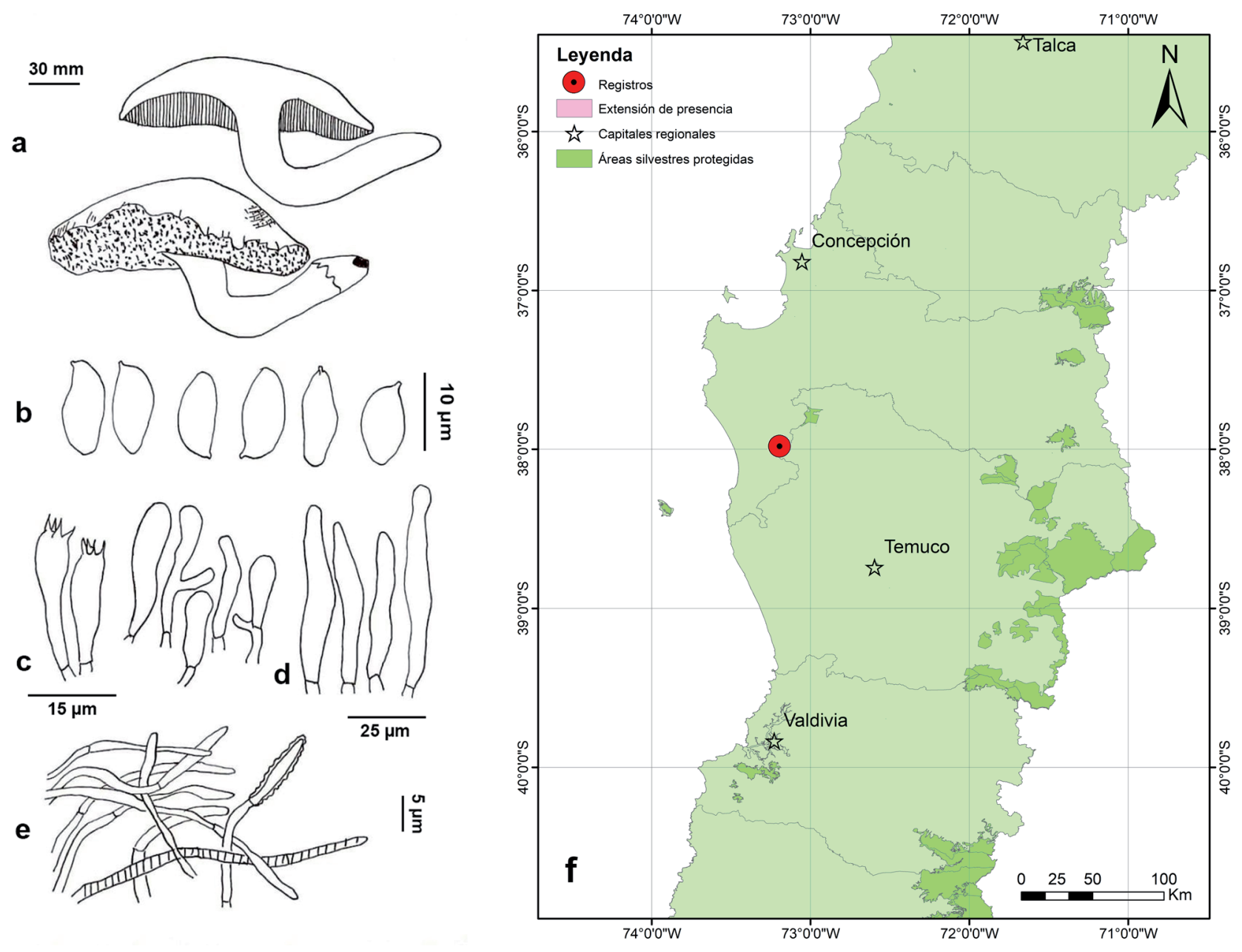

Figura 5. Boletus bresinskyanus. a. Representación de un basidioma; b. Basidiosporas; c. Basidios; d. Queilocistidios; e. Cutícula (adaptados de Garrido, 1988); f. Mapa de la distribución actual de B. bresinskyanus. 
Boletus araucariae (Fig. 6) Garrido (Sinónimo: Boletus araucarianus Garrido)

Descripción: Píleo de 75-200 x 15-40 mm, planoconvexo, pulvinado o plano, ligeramente deprimido en el centro y convexo con margen doblado, viscoso cuando está húmedo, de otro modo seco, marrón-ocre, en su mayoría sin ningún tinte marrón-rojizo, tornándose en algunos casos de tonalidades ocres hacia el margen, mate. Cutícula del píleo no extraíble. Margen enrollado durante la mayor parte del tiempo, sin extenderse. Tubos redondos alrededor del estípite, ligeramente deprimidos y levemente decurrentes, a veces asemejan una red estirada. Capa del tubo en comparación con el contexto (aproximadamente $35 \mathrm{~mm}$ de grosor), no muy gruesa (aproximadamente $10 \mathrm{~mm}$ de largo), fácilmente removible, poros angulares, bastante anchos, 3-4 por $5 \mathrm{~mm}$, amarillo-ocres mezclado con oliva-pardusco a ocre-parduzco. Tintes, marrón-ocre más oscuro, parcialmente azulados al
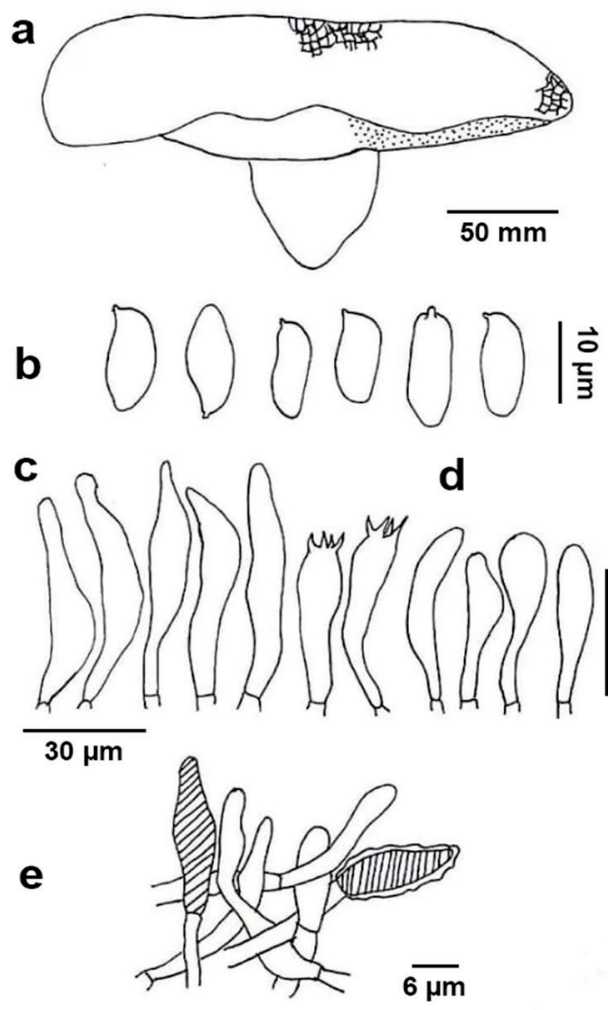

presionarlos. La capa del tubo a menudo es oblicua y el extremo superior se atenúa gradualmente. Estípite $50-60 \times 25-50 \mathrm{~mm}$, redondo, mayoritariamente amarillo vivo, a veces con manchas carmesí, en el centro ocre-pardo o verde-oliva, mitad inferior o al menos base carmesí; en algunos casos se torna carmesí-oscuro el ápice. Superficie ligeramente brillante, ápice tomentoso a la lupa. Contexto suave; margen de estípite de consistencia dura, suave en el centro, sólido, pálido-amarillento y gris-pálido.. Ausencia de micelio basal. Esporas 8.5-15 x 4.5-6 $\mu \mathrm{m}$, fusiformes a elipsoide-fusiformes, lisas, ausencia de poros, con depresión supra-hilar, pálido-ocre, parduzco. Basidios 26-45 x 8-10 $\mu \mathrm{m}$, con 4 esporas. Pleuro y queilocistidios 35-70 $\mathrm{x}$ 7-10 $\mu \mathrm{m}$, fusiformes, de color ocre-pálido a casi incoloro, de paredes delgadas. Caulocistidio 25$50 \times 6-11 \mu \mathrm{m}$, claviforme a fusiforme-claviforme. Cutícula del píleo con hifas gelatinosas cilíndricas (6-18 $\mu \mathrm{m}$ de ancho), dispuestas radialmente, de color marrón-rojizo; Células terminales afines a der-

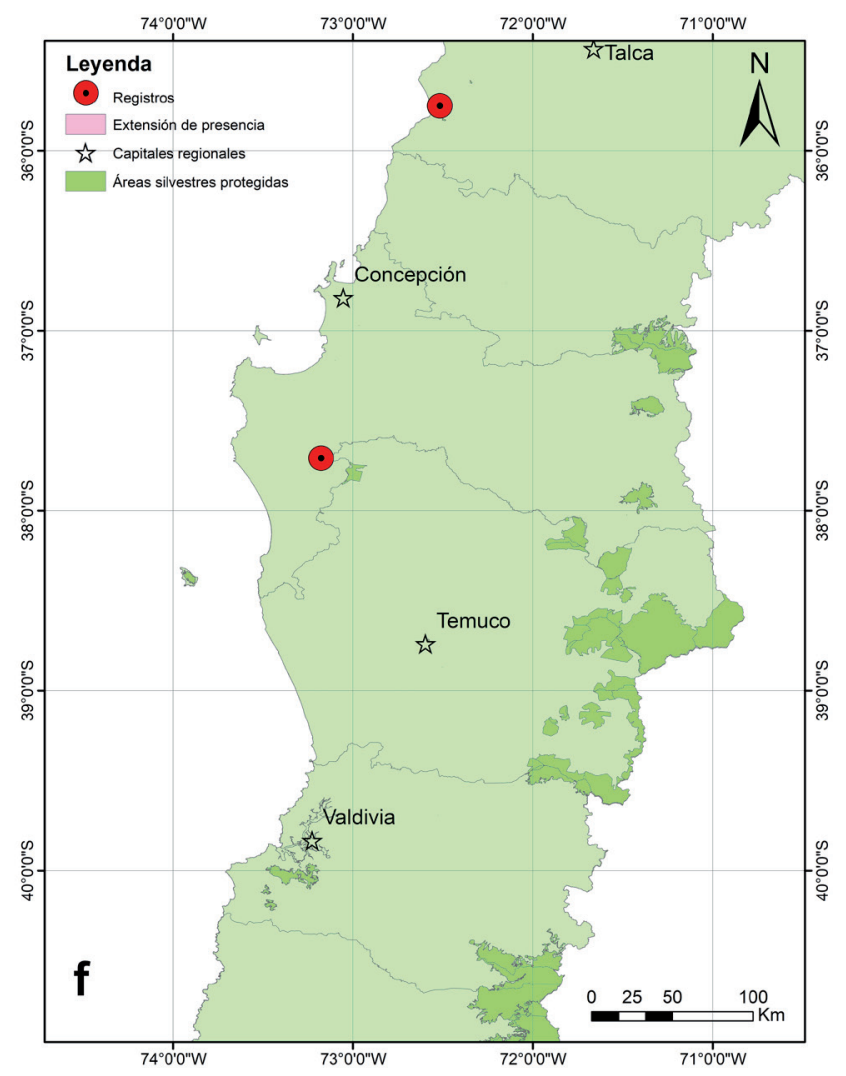

Figura 6. Boletus arancariae. a. Representación de un basidioma; b. Basidiosporas; c. Basidios, d. Queilocistidios; e. Cutícula (adaptados de Garrido, 1988); f. Mapa de la distribución actual de B. araucariae. 
matocistidios. Predominantemente fusiformes. Fí- Localidades de distribución bulas ausentes (modificado según Garrido, 1988).

Hábitat y ecología: Basidiomas en suelo bajo N. dombeyi, y Araucaria araucana (Molina) K. Koch., formando asociaciones ectomicorrícicas con Coihue (Garrido, 1988).

Distribución geográfica: Con registros en la Región del Maule y Región del Biobío.

\section{Categoría de conservación vigente en Chile: NO EVALUADA.}

Según la literatura revisada, las especies nativas del género Boletus han sido avistadas en 24 localidades, siendo la Región de los Ríos y del Biobío las más frecuentes, con 9 y 7 reportes respectivamente. A su vez, las especies más comunes de observar corresponden a $B$. loyo, $B$. loyita y $B$. putidus, mientras que $B$. bresinkyanus y $B$. araucariae sólo han sido reportadas por $\mathrm{Ga}-$ rrido (1988) en una y dos localidad respectivamente (Tabla 1).

Tabla 1. Regiones y localidades en las que se distribuye cada especie del género Boletus registrada para Chile, donde 1 indica presencia y 0 ausencia, según la literatura citada.

\begin{tabular}{cllcccccc} 
ID & Región & Localidad & B. loyo & B. loyita & B. putidus & B.chilensis & B. bresinkyanus & B. araucariae \\
\hline 1 & Maule & Vilches Alto & 1 & 0 & 1 & 0 & 0 & 0 \\
2 & Maule & Los Ruiles & 1 & 1 & 0 & 0 & 0 & 1 \\
3 & Maule & Bullileo & 1 & 0 & 0 & 0 & 0 & 0 \\
4 & Maule & Pelluhue & 0 & 1 & 0 & 0 & 0 & 0 \\
5 & Biobío & Cayumanque & 1 & 0 & 1 & 0 & 0 & 0 \\
6 & Biobío & Concepción & 1 & 1 & 1 & 0 & 0 & 0 \\
7 & Biobío & Curanilahue & 1 & 0 & 0 & 0 & 0 & 0 \\
8 & Biobío & RN Nonguén & 0 & 1 & 1 & 0 & 0 & 0 \\
9 & Biobío & Trongol Alto & 0 & 1 & 0 & 0 & 1 & 0 \\
10 & Biobío & Caramávida & 0 & 0 & 0 & 0 & 0 & 1 \\
11 & Biobío & Santa Juana & 0 & 0 & 1 & 0 & 0 & 0 \\
12 & Araucanía & Laguna Malleco & 1 & 0 & 0 & 0 & 0 & 0 \\
13 & Araucanía & Reserva Rucamanque & 1 & 1 & 1 & 0 & 0 & 0 \\
14 & Araucanía & PN Nahuelbuta & 0 & 0 & 0 & 1 & 0 & 0 \\
15 & Los Ríos & Fundo las Palmas & 1 & 0 & 0 & 0 & 0 & 0 \\
16 & Los Ríos & Cuesta Santa Elvira & 1 & 0 & 1 & 1 & 0 & 0 \\
17 & Los Ríos & Valdivia & 1 & 1 & 1 & 1 & 0 & 0 \\
18 & Los Ríos & Fundo el Junco & 1 & 0 & 0 & 0 & 0 & 0 \\
19 & Los Ríos & Los Molinos & 1 & 0 & 0 & 0 & 0 & 0 \\
20 & Los Ríos & Puerto Fuy & 1 & 0 & 0 & 0 & 0 & 0 \\
21 & Los Ríos & Cordillera pelada & 0 & 1 & 0 & 0 & 0 & 0 \\
22 & Los Ríos & La Unión & 0 & 1 & 0 & 0 & 0 & 0 \\
23 & Los Ríos & PN Alerce Costero & 0 & 0 & 1 & 0 & 0 & 0 \\
24 & Los Lagos & Popoén & 0 & 0 & 0 & 1 & 0 & 0 \\
\hline & & TOTAL & 14 & 9 & 9 & 4 & 1 & 2 \\
\hline
\end{tabular}


Finalmente, se realizó una caracterización climática y altitudinal de las especies del género Boletus presente en Chile (Figura 7), mostrando que en cuanto a la temperatura poseen rangos similares de promedio y con una amplia tolerancia térmica, desde los -3.5 hasta $\operatorname{los} 28^{\circ} \mathrm{C}$ (Fig. 7a, 7b, y 7c); sin embargo, son pocos los ejemplares que habitan en temperatura bajo cero en invierno, correspondiente a los individuos de las especies $B$. araucariae, B. loyo, B. putidus y B. chilensis que habitan en las localidades de Vilches Alto y las de la Cordillera de Nahuelbuta, zonas en las que nieva durante el invierno. En cuanto a las precipitaciones (Fig. 7d y 7e) B. araucariae es el que habita en zonas con rangos de precipitacio-
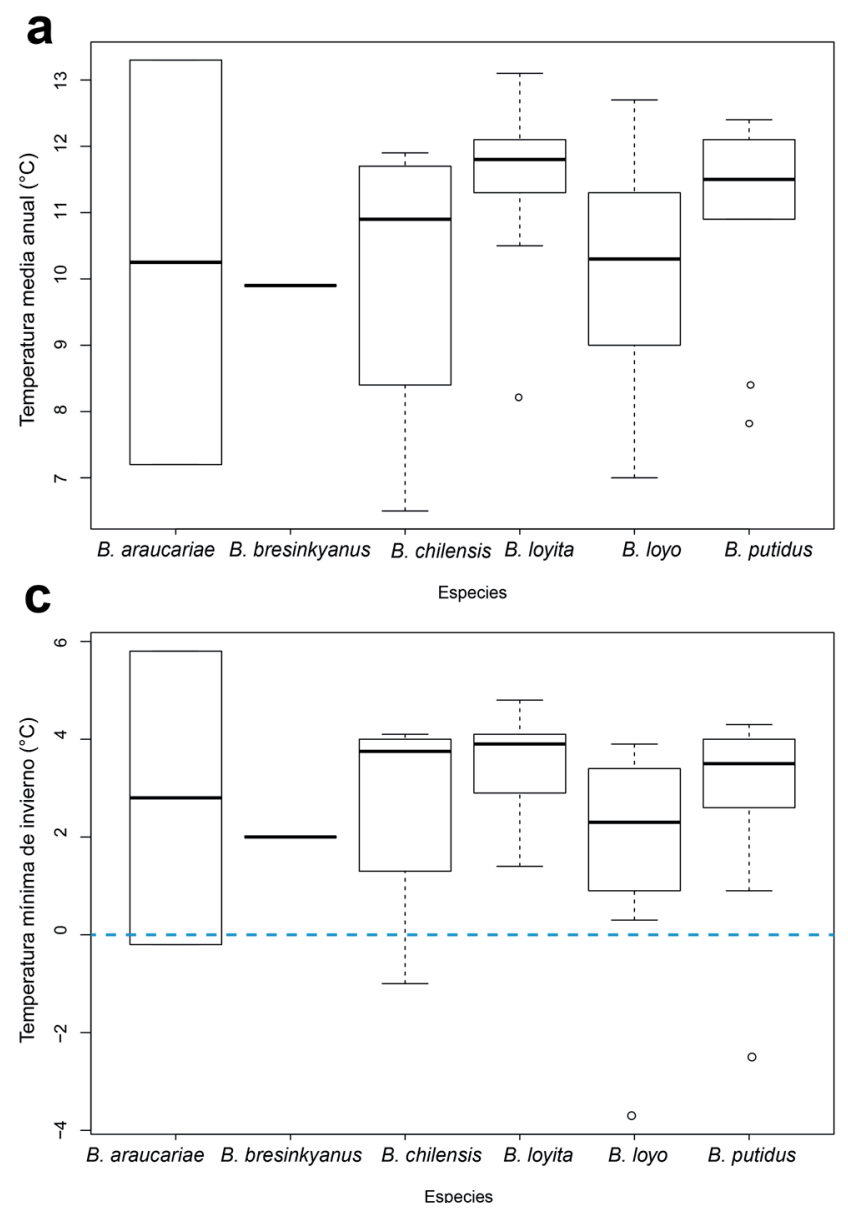

nes más bajos, entre 823 - $1550 \mathrm{~mm}$ anuales, y el que posee más alta estacionalidad en las precipitaciones, es decir, veranos más secos e inviernos más lluviosos. Por el contrario, B. chilensis es el que en promedio habita en condiciones más lluviosas y con menor estacionalidad en las precipitaciones. Las otras especies, a excepción de $B$. bresinkyanus que sólo posee un registro, habitan en rangos de precipitaciones mayores, entre $\operatorname{los} 880$ y $2.400 \mathrm{~mm}$ anuales. Finalmente, al analizar el rango altitudinal en el cual crecen las especies, la mayoría de ellos han sido reportados bajo los 1.000 m.s.n.m. (Fig. 7f), con excepción de los ejemplares que crecen en Vilches Alto y en la Cordillera de Nahuelbuta.
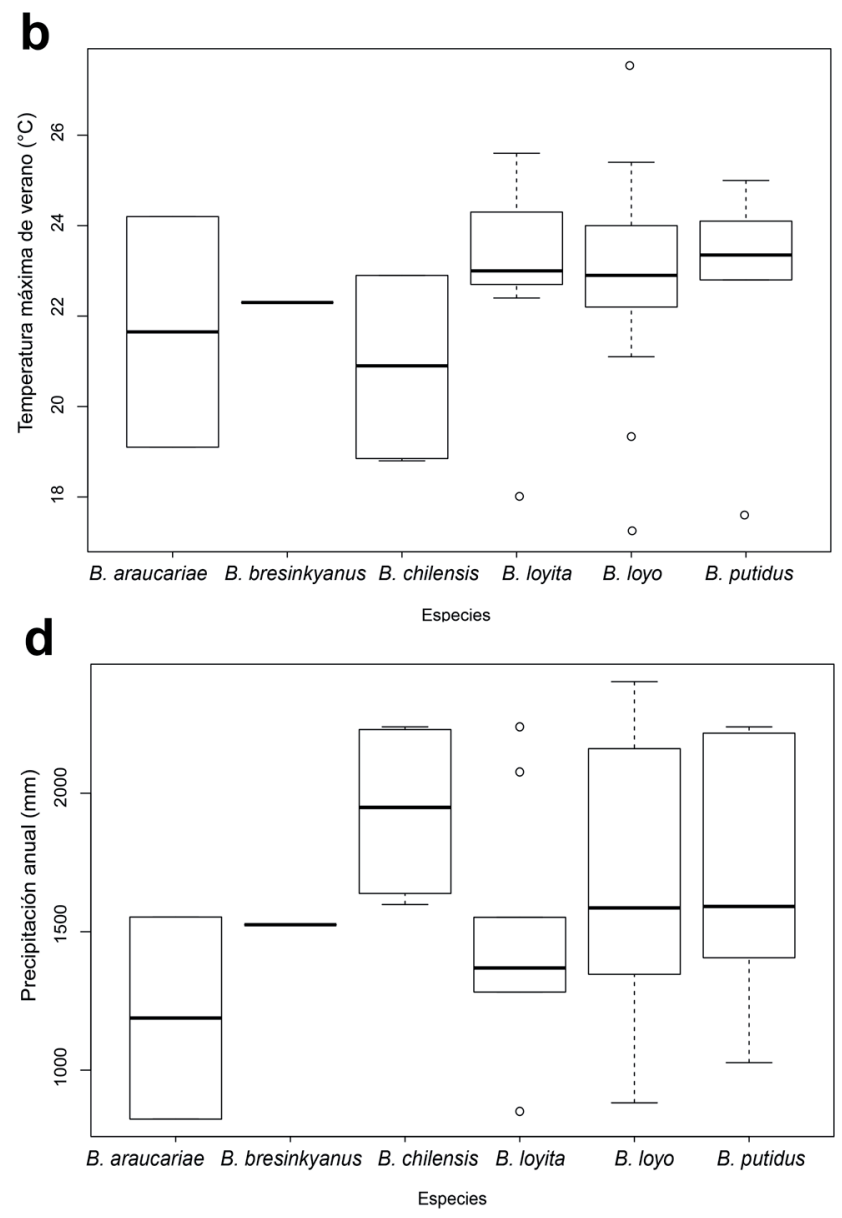

Figura 7. Caracterización climática y altitudinal para las especies del género Boletus presentes en Chile. a. Temperatura media anual $\left({ }^{\circ} \mathrm{C}\right)$; b. Temperatura máxima de verano $\left({ }^{\circ} \mathrm{C}\right)$; c. Temperatura mínima de invierno $\left({ }^{\circ} \mathrm{C}\right)$, indicando en la línea discontinua celeste la temperatura bajo $0^{\circ} \mathrm{C}$; d. Precipitación anual (mm). 

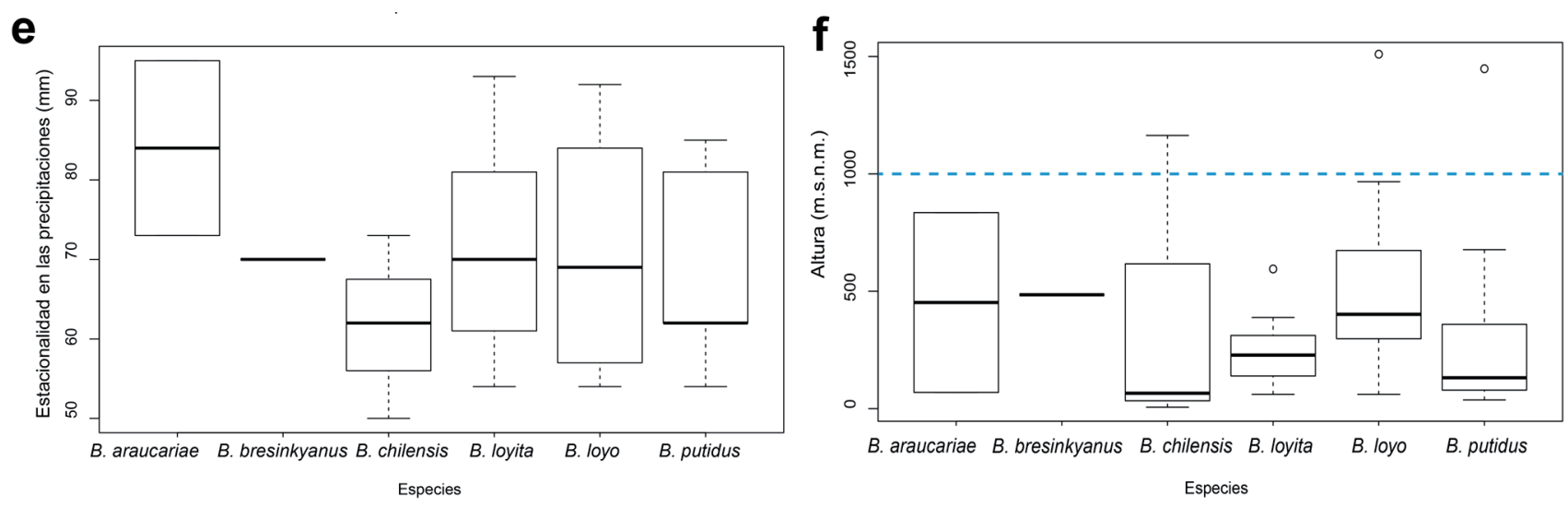

Figura 7. e. Estacionalidad de las precipitaciones (mm) y; f. Altitud (m.s.n.m.), indicando en la línea discontinua celeste el límite de 1.000 m.s.n.m.

\section{DISCUSIÓN}

Hasta la fecha, en Chile se han registrado 6 especies del género Boletus en los bosques nativos, incluyendo a B. loyo, dentro de las cuales algunas de ellas son muy similares entre sí a simple vista, llegando a confundirse si no se observan en detalle sus características microscópicas, a excepción de B. loyo el cual es distintivo por su gran tamaño y colores llamativos. En nuestro país, las especies del género Boletus poseen un rango de distribución similar, siendo el límite norte la Región del Maule, donde la Reserva Altos de Lircay es la que posee los registros más al norte de todo el género en Chile, registrándose a $B$. loyo (Garrido, 1988) y B. putidus siendo los pocos ejemplares que habitan por sobre los 1.000 m.s.n.m. y con heladas invernales. Por su parte, la distribución sur se encuentra en la Región de Los Lagos, en la localidad de Popoén (Valenzuela et al., 1998), registrándose a B. chilensis (Tabla 1). La mayoría de las especies y de los registros habitan en localidades que en invierno la temperatura no alcanza grados bajo cero (Fig. 7c), dando cuenta de su carácter más termófilo en Chile, en conjunto de sus requerimientos hídricos, que en promedio del género en Chile son de aproximadamente $1.600 \mathrm{~mm}$, donde $B$. araucariae es el que habita en zonas menos lluviosas. Esto podría explicar en parte su ausencia en los bosques de Nothofagus macrocarpa en la Región Metropolitana y Región de Bernardo O'Higgins, además de que el límite norte del género se encuentra en las zonas altas de Vilches, debido a que se acumula mayor precipitación anual.

Es importante destacar que, de las seis especies consideradas en esta revisión, cuatro de ellas se encuentran clasificadas en alguna categoría de conservación del Ministerio del Medio Ambiente. De éstas, dos se encuentran catalogadas En Peligro (EN): B. loyo y B. chilensis y una en estado Vulnerable (VU): $B$. loyita. Para $B$. loyo se propone la Categoría de Conservación EN, debido a la reducción del tamaño de la población, reducción de la población inferida o sospechada mayor o igual al 50\% en el pasado (50 años), donde la reducción, o sus causas, pueden no haber cesado. Además, de una reducción de la calidad del hábitat debido a la deforestación y niveles de explotación reales o potenciales, para consumo humano. Por otra parte, B. chilensis se encuentra EN a causa de una disminución de la calidad del hábitat deteriorada por reemplazo de vegetación nativa por plantaciones $\mathrm{y} ;$ B. loyita ha sido catalogada como VU, por una disminución de la calidad del hábitat deteriorada por aumento de frecuencia y extensión de incendios, asimismo, por contar con escasos registros. 
Hasta la fecha, B. loyo es la única especie que ha sido reportada en Argentina, específicamente en Neuquén (Spegazzini, 1912), siendo endémica de los bosques de Nothofagus del sur de Sudamérica. Por su parte, todas las otras especies del género Boletus presentes en los bosques nativos de Chile son endémicas, asociadas en su mayoría a $N$. obliqua y $N$. dombeyi, donde $B$. loyo se asocia además a $N$. glauca y $N$. alpina, mientras que $B$. loyita se asocia también a $N$. alpina, N. glauca y N. alessandrii, siendo este último catalogado En Peligro y Rara (ENR) según la Clasificación del Ministerio del Medio Ambiente. Por su parte, $B$. araucariae y $B$. bresinkyanus poseen un elevado grado de endemismo restringido, donde $B$. araucariae ha sido documentado únicamente en bosques de N. dombeyi y Araucaria araucana en el sector de Caramávida, la cual es una zona de la Cordillera de Nahuelbuta no protegida por el SNASPE, mientras que $B$. bresinkyanus ha sido documentado en el sector de Trongol Alto, también perteneciente a la Cordillera de Nahuelbuta, además de la Reserva Nacional Los Ruiles. De este modo, la zona de la Cordillera de Nahuelbuta ubicada en la Región del Biobío cobra un elevado valor de conservación en cuanto al género Boletus, ya que alberga 4 de las 6 especies, incluyendo $B$. loyo y B. loyita. Es importante destacar que todas las especies descritas son comestibles, lo que hace aún más necesaria su conservación ya que conforman parte de los Productos Forestales No Madereros (PFNM). Según Salas et al., (2006) los bosques de $N$. obliqua del sur de Chile al igual que otros bosques nativos dominados por otras especies arbóreas, han sufrido un proceso de degradación acelerado por la expansión de los terrenos de cultivo, incluyendo monocultivos, lo que afecta directamente a las especies de hongos asociados a estos bosques, por lo que es de suma importancia proteger estos ecosistemas donde habitan hongos nativos de gran importancia ecosistémica, económica y social, como es el caso de los hongos silvestres comestibles altamente recolectados para consumo y comercialización, en los que destacan las especies B. loyo, B. loyita y B. chilensis. Además, Alarcón \& Cavieres (2015) realizaron estudios de cómo el cambio climático afectaría a los bosques dominados por Nothofagus, indicando que se estima que las especies de plantas con nichos amplios se verán menos afectadas a lo largo de gradientes ambientales, pero además se espera que migren a latitudes y altitudes mayores. Este escenario podría ser un riesgo para el género Boletus debido a que, si sus hospederos se desplazan a mayores altitudes, donde además el clima es más frío; podrían verse afectadas negativamente las poblaciones de Boletus, sin embargo, es necesario realizar más estudios con mayor cantidad de registros para poder evaluar el efecto del cambio climático sobre este género.

Finalmente, los autores quieren destacar que existe una especie de la familia Boletaceae endémica de Chile inconfundible por su morfología, aspecto secotoide creciendo semienterrado y su coloración ocráceo-amarillento, Gastroboletus valdivianus E. Horak, que se distribuye entre la Región del Biobío y Región de Los Ríos, siendo la única especie conocida del género en Sudamérica (Horak, 1977) y se encuentra catalogada actualmente En Peligro (EN) (MMA). Esta especie no fue incluida en esta revisión, por pertenecer a un género distinto de Boletus.

\section{AGRADECIMIENTOS}

Los autores agradecen al Prof. Dr. emer. Reinhard Agerer y a la Dra. Patricia Silva-Flores por su colaboración en la traducción del idioma alemán al español de las especies $B$. bresinkyanus y $B$. araucariae. A Valentina Cáceres por colaborar con las ilustraciones de las especies 
descritas. A Eitel Thielemann, Gabriel Cartés y Johan Andrianoff por facilitarnos las fotografías macroscópicas. Al Dr. Götz Palfner por su activa participación en la incorporación de fichas de clasificación de especies de macrohongos en el Ministerio del Medio Ambiente dentro de las cuales se encuentran 4 de las especies consideradas en esta revisión.

\section{REFERENCIAS}

Alarcón, D., \& L. Cavieres. (2015) In the right place at the right time: habitat representation in protected areas of South American Nothofagusdominated plants after a dispersal constrained climate change scenario. PloS one, 10(3), e0119952.

Corner, E. (1972) Boletus in Malaysia. Government Printing Office, Singapore, Malaya. 263 pp.

Dentinger, B. T., Ammirati, J. F., Both, E. E., Desjardin, D. E., Halling, R. E., Henkel, et al. (2010) Molecular phylogenetics of porcini mushrooms (Boletus sect. Boletus). Molecular Phylogenetics and Evolution, 57(3), 1276-1292.

Donoso, C. (1990) Ecología Forestal, El Bosque y su Medio Ambiente. Editorial Universitaria, ISBN 956-11-0167-9.

Garrido, N. (1985) Index Agaricalium Chilensium. Bibliotheca Mycologica 99, J. Cramer, Vaduz, ISN 3-7682-1435-10.

Garrido, N. (1988) Agaricales und ihre mykorrhizen in den Nothofagus-Waldem mittelchiles. Bibliotheca Mycologica. Tomo 120, J. Cramer, Berlín, Stuttgart, Alemania, 528 pp.

Hall, I.R., Lyon, A., Wang, Y. \& L. Sinclair. (1998) Ectomycorrhizal fungi with edible fruiting bodies - 2. Boletus edulis. Econ. Bot. 52, 44-56.

Hijmans, R.J., Cameron, S.E., Parra, J.L., Jones, P.G. \& A. Jarvis. (2005) Very high resolution interpolated climate surfaces for global land areas. International Journal of Climatology, 25, 1965-1978.
Horak, E. (1977) New and rare boletes from Chile. Bol. Soc. Arg. Bot. 18(1-2): 97-109.

Horak, E. (2005) Röhrlinge und Blätterpilze in Europa. 6. Auflage. - München: Elsevier. 555 Seiten, 48 Abb. auf 6 Tafeln. ISBN 3-8274-1478-4, Euro 40.

Horak, E. (2011) Revision of Malaysian Species of Boletales s.l. (Basidiomycota) Described by E.J.H. Corner (1972, 1974). Malayan Forest Records. 51:1-283.

Kirk, P. M., Cannon, P. F., David, J. C., \& J. A. Stalpers. (2008) Ainsworth and Bisby's dictionary of the fungi. Wallingford. International Mycological Institute.

Marín, C., Torres, D., Furci, G., Godoy, R., \& Palfner, G. (2018). Estado del arte de la conservación del reino Fungi en Chile. Biodiversidata 7-98-115.

Murrill, W. A. (1909) The Boletaceae of North America-I. Mycologia, 1(1), 4-18.

Palfner, G. (2005). Tylopilus temucensis sect. Oxydabiles (Fungi, Basidiomycota, Boletaceae), new species and first record of the genus from South American Nothofagus forest. Fungal Diversity, 20, 157-166.

Piepenbring, M. (2015) Introducción a la Micología en los Trópicos. St. Paul, Minnesota: The American Phytopathological Society. 366 pp. 
Salas, C., LeMay, V., Núñez, P., Pacheco, P. \& A. Singer, R. (1986) The Agaricales in Modern TaxoEspinosa. (2006) Spatial patterns in an old-growth nomy. Koenigstein, Germany: Koeltz Scientific Nothofagus obliqua forest in south-central Chile. Books. $981 \mathrm{pp.}$

Forest ecology and management, 231(1-3), 38-46.

Smith, A. \& H. Thiers. (1971) The boletes of Michigan. University of Michigan Press, Ann Arbor.

Spegazzini, C. (1912) Mycetes argentinenses. VI. Anales Mus. Nac. Buenos Aires, 23: 167-244

Valenzuela, E., Moreno, G., Garnica, S. \& C. Singer, R. (1965) Die Pilze Mitteleuropas. Band V: die Röhrlinge Teil I: die Boletaceae (ohne Boletoideae). Verlag Julius Klinkhardt, Bad Heilbrunn.

Singer, R. (1967) Die Pilze Mitteleuropas. Band VI: Die Röhrlinge Teil II: die Boletoideae und Strobilomycetaceae. Verlag Julius Klinkhardt, Bad Heilbrunn.

Ramírez. (1998) Micosociología en bosques nativos de Nothofagus y plantaciones de Pinus radiata en la X Región de Chile: diversidad y rol ecológico. Revista Chilena de Historia Natural, 71, 133-146.

Watling, R. (1970) British fungus flora Volume I: Boletaceae, Gomphidiaceae, Paxillaceae. Royal Botanic Garden, Edinburgh. 